\title{
Calibration Technology and Application of Ultrasonic Sensor for Partial Discharge Detector
}

\author{
Guangke $\mathrm{Xu}^{1, \mathrm{a}^{*}}$, Fuchun Sun ${ }^{1, \mathrm{~b}}$, Weiwei Zhang ${ }^{1, \mathrm{c}}$, Ming $\mathrm{Li}^{1, \mathrm{~d}}$, \\ Zhenhua Zhu ${ }^{1, \mathrm{e}}$, Andong Wang ${ }^{1, \mathrm{f}}$, Fuqiang Zhao ${ }^{1, \mathrm{~g}}$, Jie Liu $^{2, \mathrm{~h}}$, \\ and Xiuzhong Gong ${ }^{3, i}$ \\ ${ }^{1}$ State Grid Shandong Electric Power Research Institute, Jinan, 250002, China \\ 2 State Grid Yantai Power Supply Company, Yantai, 264000, China \\ ${ }^{3}$ Shandong Zhongshiyitong Group Co., Ltd. Jinan, 250002, China \\ axuguangkesd@163.com, bleaftongtong@126.com, cxuleaff@163.com, d13668816013@163.com,

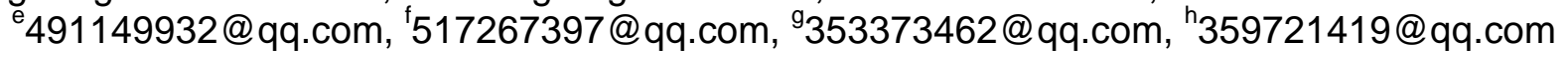

Keywords: ultrasonic, sensor, detector, partial discharge, calibration technology.

Abstract. Ultrasonic partial discharge detector can discover and locate the partial discharge, and is used widely. Recently more and more ultrasonic sensor and partial discharge detector were produced by different producer. There are so many styles and kinds that detector functions are intermingled. For example, center frequency, frequency range, sensitivity are different widely, making the detect results are highly varied. So it is necessary strongly to standard the calibration technology and start application of ultrasonic partial discharge detector. The paper introduces the method and application for ultrasonic partial discharge detector calibration.

\section{Introduction}

Insulation material for electric apparatus used in high voltage and strong electric field condition will deteriorate progressively, because of discharge, electromagnetic force, thermal stress, and so on. So partial discharge will appear at strong electric field locations, and it's the symptom of insulation defects.

Ultrasonic partial discharge detector can discover and locate the partial discharge using the ultrasonic sensor receiving the ultrasonic signals produced by partial discharge in GIS or transformer. Ultrasonic partial discharge detector can detect wide frequency range, from $20 \mathrm{kHz}$ to $200 \mathrm{kHz}$. Besides, ultrasonic method is unauthoritativeness, so it has better capacity of resisting disturbance and higher sensitivity. Because of the above excellence, ultrasonic partial discharge detector is widely used to detect partial discharge in GIS and transformer in power system and obtain good results.

With the development of science and technology and the progress of society, more and more ultrasonic sensor and partial discharge detector were produced by different producer. There are so many styles and kinds that detector functions are intermingled. For example, center frequency, frequency range, sensitivity are different widely, making the detect results are highly varied. So it is necessary strongly to standard and start the calibration technology and application of ultrasonic partial discharge detector. The paper introduces the method and application for ultrasonic partial discharge detector calibration.

\section{Detector theory of ultrasonic partial discharge detector}

When partial discharge appearing, numerator will strike each other strongly, and insulation gets bigger in a moment because of heat. All the elements will appear impulse of pressure wave in macroscopic phenomenon, ultrasonic wave is one of frequencies are bigger than $20 \mathrm{kHz}$. At the moment of partial discharge, the location of discharge can be seen a pulse signal spot, sound wave will disseminate all around in the shape of spherical wave, conforming to the laws of mechanical wave propagation. It disseminates with different speed at different medium and generates reflex and 
refraction at medium boundary. Ultrasonic sensors installed at external part of equipment can detect partial discharge signals by changing ultrasonic signals to electric signals, filtering, amplifying and so on.

System structure of ultrasonic partial discharge detector is shown in Fig. 1. It is made up by ultrasonic sensor, preamplifier, filter, post-detector, digital processing oscilloscope, the final signals can be seen in the computer directly.

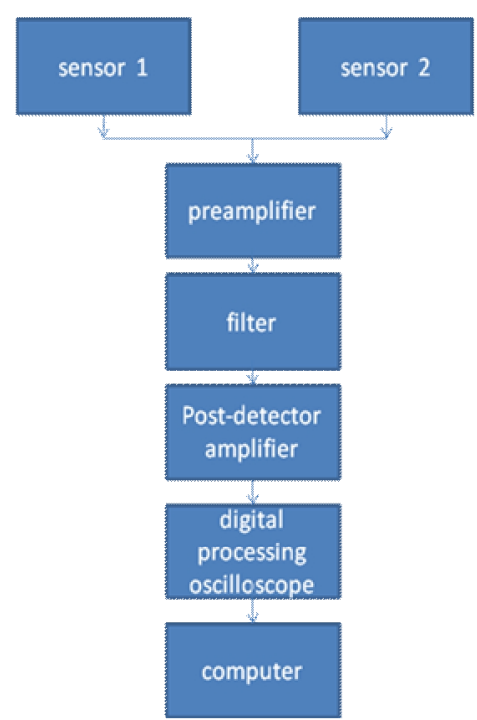

Fig. 1 system structure of ultrasonic partial discharge detector

Company standard of State Grid "Technical Regulations of Ultrasonic Partial Discharge Detector" was formulated recently. It formulates the technical regulations of ultrasonic partial discharge detector.

(1) Sensitivity of ultrasonic sensor: Sensitivity is the response to ultrasonic signals. It's one of the important performance criteria, and can be shown in $\mathrm{V} /(\mathrm{m} / \mathrm{s})$. Technical Regulations demands: peak sensitivity of ultrasonic sensor is higher than $60 \mathrm{~dB}(\mathrm{~V} /(\mathrm{m} / \mathrm{s}))$ and average sensitivity is higher than $40 \mathrm{~dB}(\mathrm{~V} /(\mathrm{m} / \mathrm{s}))$.

(2) linearity error: is less than $\pm 20 \%$.

\section{Calibration technology of ultrasonic partial discharge detector}

(1) Calibration of ultrasonic sensor sensitivity

JJF 1337-2012 《Calibration Specification for Acoustic Emission Sensors (Comparative Method)》 shows the calibration method for the ultrasonic sensor sensitivity. The measurement standard needed are shown in the following.

1) Test block

The test block is made by steel. It's diameter is more than $400 \mathrm{~mm}$ and height is more than $180 \mathrm{~mm}$. The two faces of the block is smooth and parallel, and depth of parallelism is less than $0.12 \mathrm{~mm}$. The block is detected by longitudinal wave at $2.5 \mathrm{MHz}$ and the returning wave is less than $10 \%$ of the first wave.

2) Signal generator

Signal generator could produce narrow pulse signals which pulse width is less than 1 us and amplitude is more than $5 \mathrm{~V}$.

3) Acoustic emission transducer

Acoustic emission transducer has wide frequency range or resonance frequency is more than 1 $\mathrm{MHz}$.

4) Reference sensor

The reference sensor should be calibrated periodically.

Calibration methods are shown in the following: 
The connection figure of calibration methods is shown in Fig. 2. The acoustic emission transducer is located at the centre of one face of the test block, and the reference sensor and the calibrated sensor are located at the center of the other face successively. The signal generator produces the same narrow pulse signals and records the response voltage waves of reference and the calibrated sensors respectively.

FFT is used to calculate the amplitude response $\mathrm{U} 1(\mathrm{fm})$ and $\mathrm{U} 2(\mathrm{fm})$ of reference and the calibrated sensors with fm from $100 \mathrm{kHz}$ to $1 \mathrm{MHz}$. About $10 \mathrm{kHz}$ is recommended to consider as the frequency blank for FFT. The frequency response and sensitivity of reference sensor are gained from the higher level calibration and calibrated sensor is calculated from formula (1).

$$
\mathrm{S}_{2}\left(f_{m}\right)=\frac{\mathrm{U}_{2}\left(f_{m}\right)}{\mathrm{U}_{1}\left(f_{m}\right)} \mathrm{S}_{1}\left(f_{m}\right)
$$

In the above formula (1):

$\mathrm{S}_{1}\left(f_{\mathrm{m}}\right)$ - sensitivity of reference sensor at $f_{\mathrm{m},} \mathrm{V} /\left(\mathrm{m} \cdot \mathrm{s}^{-1}\right)$;

$\mathrm{S}_{2}\left(f_{\mathrm{m}}\right)$ - sensitivity of calibrated sensor at $f_{\mathrm{m},} \mathrm{V} /\left(\mathrm{m} \cdot \mathrm{s}^{-1}\right)$;

$\mathrm{U}_{1}\left(f_{\mathrm{m}}\right)$ - amplitude response of reference sensor at $f_{\mathrm{m}}, \mathrm{V}$;

$\mathrm{U}_{2}\left(f_{\mathrm{m}}\right)$ - amplitude response of calibrated sensor at $f_{\mathrm{m}}, \mathrm{V}$.

Thus integral sensitivity and frequency response curve can be drawn by recording all the calculated points.

(2) Linearity error test

Linearity error test will be done as Fig. 3. Make the signal generator produce sinusoidal signals and select frequency response to the highest sensitivity. Adjust the signal generator amplitude and amplifier gain to make the ultrasonic partial discharge detector get plateful approximately. Record the detector reading Amax and generator amplitude Umax. Then adjust the generator to $\lambda^{*} \operatorname{Umax}(\lambda=0.8 、 0.6 、 0.4$ 、 0.2 ) and the detector reading $A \lambda$ successively. The linearity error can be calculated with formula (2).

$$
\delta_{i}=\frac{\mathrm{A}_{\lambda}-\lambda \cdot \mathrm{A}_{\max }}{\lambda \cdot \mathrm{A}_{\max }} \times 100 \%
$$

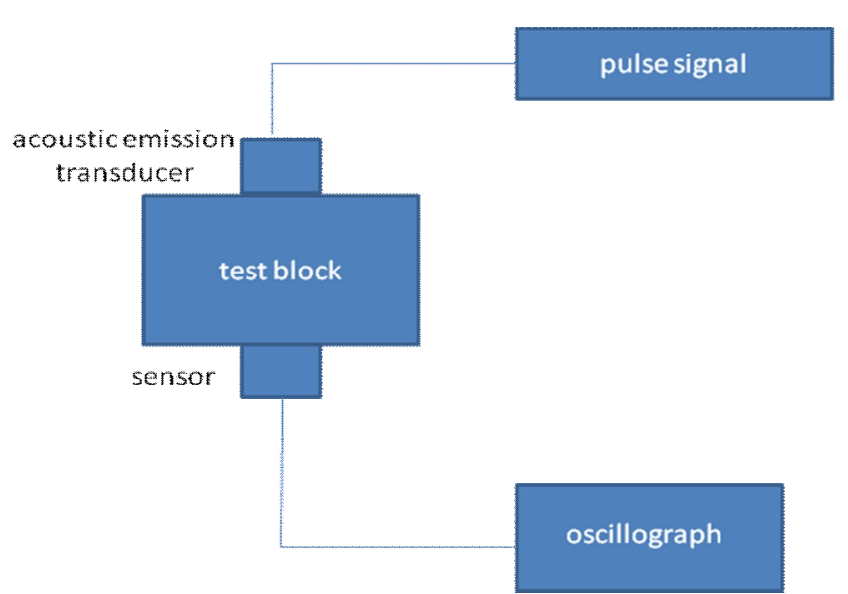

Fig. 2 The connection figure of calibration methods

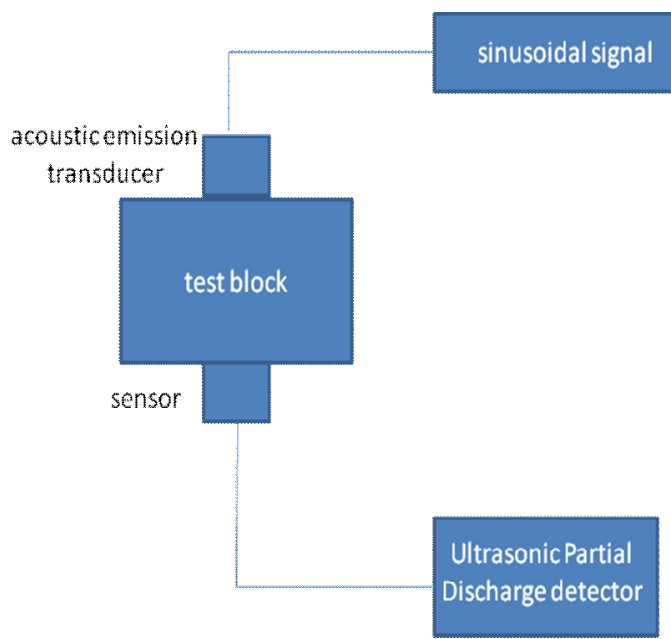

Fig. 3 Linearity error test

\section{Calibration results}

Select an ultrasonic partial discharge detector with frequency range from $20 \mathrm{kHz}$ to $200 \mathrm{kHz}$. Sensitivity curve is drawn as Fig. 4. Conclusions can be got that the highest and average sensitivity of the sensor are correspond to technical regulations. 


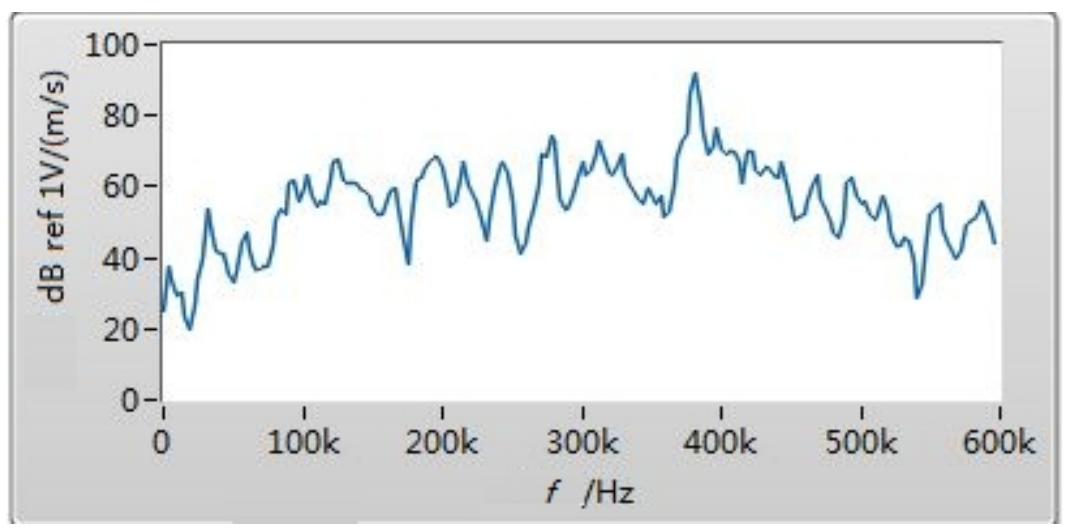

Fig. 4 Sensitivity curve of an ultrasonic partial discharge detector The linearity error test result is shown in Table 1.

Table 1 linearity error test result

\begin{tabular}{|c|c|c|c|c|}
\hline$\lambda$ & $\begin{array}{c}\text { signal } \\
\text { amplitude/ }\end{array}$ & signal frequency/kHz & $\mathrm{A}_{\lambda} / \mathrm{dB}$ & linearity error $/ \%$ \\
\hline 1 & 10 & 100 & 49 & 0 \\
\hline 0.8 & 8 & 100 & 47 & -0.71 \\
\hline 0.6 & 6 & 100 & 45 & 5.16 \\
\hline 0.4 & 4 & 100 & 42 & 11.67 \\
\hline 0.2 & 2 & 100 & 36 & 11.94 \\
\hline
\end{tabular}

In the table, the biggest linearity error of all the points is $11.94 \%$ less than $20 \%$.

\section{Summary}

The paper summarizes the structure feature and technical regulations, introduces a practicable calibration method in the lab. And the author selects an ultrasonic partial discharge detector to calibrate, and verify the detector is corresponding to technical regulations. So ultrasonic partial discharge detector calibration is practicable and necessary. It can detect the function of detector, and ensure the results reliable and accurate, and safeguard the power net safety.

\section{References}

[1] Y. Cheng, X. Xie, Y. Chen, X. Hu and Z. Zhu, Proceedings of the CSEE, Vol. 20, pp. 99-102, 2004

[2] JJF 1337-2012 《Calibration Specification for Acoustic Emission Sensors (Comparative Method)》

[3] Y. Qiu, High Voltage Apparatus, Vol. 33, pp. 36-40, 1997.

[4] N Yang, Electrical Measurement and Instrumentation, Vol 51, pp. 104-109. 